\title{
Auditory Temporal Processing as a Specific Deficit Among Dyslexic Readers
}

\begin{abstract}
Leah Fostick
Ariel University Center of Samaria, Ariel, Israel

\author{
Sharona Bar-El, Ronit Ram-Tsur \\ Bar-Ilan University, Ramat-Gan, Israel
}

The present study focuses on examining the hypothesis that auditory temporal perception deficit is a basic cause for reading disabilities among dyslexics. This hypothesis maintains that reading impairment is caused by a fundamental perceptual deficit in processing rapid auditory or visual stimuli. Since the auditory perception involves a number of mechanisms and temporal processing is only one of them, in the current paper, we tested, in addition to auditory temporal processing, also auditory intensity and spectral processing among dyslexic and normal readers. In addition, we examined whether poor performance of dyslexic readers in auditory temporal processing tasks results from a difficulty in working memory, by testing differences in auditory processing, controlling for working memory. Thirty-seven adult dyslexic readers and 40 adult normal readers performed a battery of tests measuring auditory temporal processing (gap detection and dichotic temporal order judgment), auditory intensity processing (absolute threshold and intensity discrimination), auditory spectral processing (spectral temporal order judgment) and working memory (backward digit span). Performance on auditory spectral and temporal processing task was poorer among dyslexic readers, as compared to normal readers, even after controlling for working memory, but no difference was found in intensity processing tasks. These results suggest that dyslexic readers exhibit a specific deficit in auditory temporal processing, which cannot be attributed either to general perceptual deficit in auditory processing, or to working memory.
\end{abstract}

Keywords: dyslexia, auditory temporal perception, auditory intensity perception, auditory spectral perception, working memory

\section{Background}

DD (developmental dyslexia) is defined as a specific functional failure to acquire age-appropriate reading skills in otherwise normally developing children (Curtin, Manis, \& Siedenberg, 2001; Stanovich, 1988; Vellutino, 1979). Although there is a consensus that DD (in which reading retardation is a major manifestation) is a neurological disorder, the underlying biological and cognitive causes of the reading retardation are still extensively debated (Ramus, 2003). Studies exploring the core deficit of dyslexia have led to different theories regarding the main cause of dyslexia, such as difficulties in phonology (Snowling, Bishop, \& Stothard, 2000), visual and auditory temporal perception (Stein, 2001; Tallal, 1980), attention (Facoetti, Paganoni, Turatto, Marzola, \& Mascetti, 2000) and memory (Swanson \& Jerman, 2007).

The present study will focus on examining the hypothesis that auditory temporal perception deficit is a

Leah Fostick, Department of Communication Disorders, Ariel University Center of Samaria.

Sharona Bar-El, School of Education, Bar-Ilan University.

Ronit Ram-Tsur, School of Education, Bar-Ilan University. 
basic cause for reading disabilities among dyslexics. This hypothesis maintains that reading impairment is caused by a fundamental perceptual deficit in processing rapid auditory or visual stimuli (Ben-Artzi, Fostick, \& Babkoff, 2005; Tallal, 1980). Speech, which is composed of brief stimuli presented rapidly, is especially vulnerable to deficit in temporal processing. A temporal processing impairment would reduce the ability of the individual to accurately perceive critical elements in the speech stream. This inability, in turn, disrupts the establishment of a stable phonological code (Ben-Artzi et al., 2005; Chung, McBride-Chang, Wong, Cheung, Penney, \& Ho, 2008; Meyler \& Breznitz, 2005; Tallal \& Piercy, 1973a). Consequently, most of the studies examining the auditory temporal perception deficit employ different tasks measuring temporal processing, such as TOJ (temporal order judgment) (Ben-Artzi et al., 2005; Chung et al., 2008; Reed, 1989; Tallal, 1980; Tallal \& Piercy, 1973a, 1973b); backward masking (Ramus et al., 2003); gap detection (Van Ingelghem, van Wieringen, Wouters, Vandenbussche, Onghena, \& Ghesquiere, 2001); and categorical perception of phonemes and non-speech analogues (Breier et al., 2001; Reed, 1989; Serniclaes, Sprenger-Charolles, Carre, \& Demonet, 2001). These tasks involve the perception of auditory information over a short period of time. The cue for completing these tasks is the ability to correctly perceive the rapidly changing stimuli. The findings that dyslexic readers perform poorly on these tasks as compared to normal readers served to support the hypothesis that auditory temporal perception deficits are the basis for dyslexia.

However, since the auditory perception involves a number of mechanisms and temporal processing is only one of them, a question may be legitimately asked as to whether dyslexia originates from a deficit in temporal processing, or whether dyslexic readers' deficit in processing auditory stimuli does not reflect the core deficit, but rather represents a more fundamental deficit in auditory perception related also to other mechanisms of auditory perception. Many of the studies testing auditory perception among dyslexic readers have used tasks which involve tones that differ in frequency (Ben-Artzi et al., 2005; Breier et al., 2001; Murphy \& Schochat, 2009; Nagarajan, Mahncke, Salz, Tallal, Roberts, \& Merzenich, 1999; Reed, 1989; Tallal, 1980; Tallal \& Piercy, 1973a, 1973b). It has been suggested in previous studies that these tasks measure spectral perception, in addition to temporal perception. Therefore, poor performance on these tasks could result from a failure to accurately process spectral cues rather than temporal cues (Ben-Artzi et al., 2005; Fostick \& Babkoff, 2010).

Another component of auditory processing is intensity perception, which involves the ability to discriminate between the loudness of different tones. This type of processing has not been tested often among dyslexic readers, when the tested has yielded inconsistent results. In some studies, dyslexic readers performed similarly to normal readers on intensity discrimination tasks (Ahissar, Protopapas, Reid, \& Merzenich, 2000; Kujala, Lovio, Lepistö, Laasonen, \& Näätänen, 2006), while on others, reading impaired children were found to be poorer than normal readers (Cacace, McFarland, Ouimet, Schrieber, \& Marro, 2000; Fischer \& Hartnegg, 2004; McArthur \& Hogben, 2001). Thus, in the current study, we propose to test whether auditory temporal processing deficit found in dyslexic readers reflects a specific deficit in the temporal processing mechanism, or whether it reflects a wider deficit in auditory processing, which also involves spectral and intensity perception.

Additional problem has been raised in interpreting the data as supporting the auditory temporal hypothesis of dyslexia. It has been suggested that the poor performance of dyslexic readers in auditory temporal processing tasks results from a difficulty in working memory which serves as a bottleneck in performing the auditory temporal processing tasks (Ahissar, 2007; Banai \& Ahissar, 2006). Working memory refers to the ability to process and manipulate ongoing information, in order to use it for continuous behavior (Baddeley, 2003). Baddely (2003) hypothesized that the working memory includes two systems of encoding sound-based (the 
phonological loop) and visual- and spatial- based information (the visuo-spatial sketchpad), as well as a generating system (the central executive). This latter system integrates the information received through the phonological loop and the visuo-spatial sketchpad with already stored information, while controlling and monitoring attention. According to the hypothesis, the primary function of phonological short-term memory is to support the long-term learning of the phonological structure of the language (Baddeley, Gathercole, \& Papagno, 1998; Gathercole, Tiffany, Briscoe, \& Thorn, 2005). Therefore, inadequate short-term memory may cause difficulties in learning the sound structure of new words (Gathercole et al., 2005). It was hypothesized that dyslexic readers have difficulties in the phonological loop and the central executive, but not in the visuo-spatial sketchpad (Archibald \& Gathercole, 2006; Jeffries \& Everatt, 2004). Additional studies have shown that dyslexic readers do have difficulties in working memory as compared with normal readers (Chung, Ho, Chan, Tsang, \& Lee, 2010; De Clercq-Quaegebeur, Casalis, Lemaitre, Bourgois, Getto, \& Vallée, 2010; Laasonen, Leppämäki, Tani, \& Hokkanen, 2009).

Taking into account these findings, the present study focused on two objectives: The first one is to test whether the shown auditory temporal processing deficit among dyslexic readers is specific to the perception of temporal processing, or whether it reflects a more general deficit in auditory processing which may include spectral and intensity processing as well; The second is that will the deficit in auditory processing exhibited by dyslexic readers be evident also after controlling for working memory. According to the auditory temporal processing deficit theory for dyslexia, we hypothesize that adult dyslexic readers will show deficit relative to normal readers on auditory temporal processing. Furthermore, we posit that the deficit in the auditory temporal processing will be independent of working memory ability. Therefore, we hypothesize poorer performance for dyslexic readers on auditory temporal processing tasks, even when statistically controlling for working memory.

\section{Method}

\section{Participants}

Thirty-seven students diagnosed with dyslexia (23 men, 14 women) and 40 normal adult readers (25 men, 15 women), aged 18 to 35 participated in the study. Diagnosis of dyslexia was based on an official diagnosis from a known diagnostic authority and on a diagnostic battery of reading and phonological tests that approved the initial diagnosis and demonstrated significantly poorer reading ability and phonological awareness among the dyslexic groups (see Table 1). The participants were screened for normal hearing and were native Hebrew speaking university or college students. All participants completed all the study battery.

\section{Diagnosing Battery}

Reading regular words (Shatil, 1995a). Hebrew written language has both deep and shallow orthographies. In shallow orthography, the words are dotted creating high spelling-to-sound correspondence, and in deep orthography, the words are undotted, creating a low spelling-to-sound correspondence (Frost, 1994). In this task, we used reading regular and undotted words (For a detailed explanation about punctuation in Hebrew, see Ram-Tsur, Faust, \& Zivotofsky, 2008). This task was designed to measure the amount of correct words per minute the participant is able to read. The participant was presented with a list of 217 solitary undotted words. The words included low and high frequency words, words with different lengths (three to seven letters), verbs and nouns in different declensions and homographs (i.e., words that can be read differently 
without punctuation). Each participant was asked to read the words as fast and correct as he can during one minute. The score reflects the number of words per minute the participant read correctly (Shatil, 1995a).

Table 1

Means and SDs of Accuracy and Reaction Time of Dyslexia Diagnostic Tests for Dyslexic and Normal Readers

\begin{tabular}{|c|c|c|c|c|c|}
\hline \multirow[t]{2}{*}{ Task } & \multicolumn{2}{|c|}{ Dyslexic readers } & \multicolumn{2}{|c|}{ Normal readers } & \multirow[t]{2}{*}{$F$} \\
\hline & Mean & $S D$ & Mean & $S D$ & \\
\hline Reading regular words $^{1}$ & 80.38 & 28.25 & 125.70 & 15.78 & $77.03^{* * *}$ \\
\hline Reading non-words ${ }^{1}$ & 70.70 & 9.36 & 33.35 & 13.00 & $211.56^{* * *}$ \\
\hline \multicolumn{6}{|l|}{ Phoneme deletion } \\
\hline Accuracy $^{1}$ & 16.43 & 3.01 & 19.43 & 0.93 & $35.97^{* * *}$ \\
\hline Reaction time ${ }^{2}$ & 97.59 & 28.06 & 61.15 & 8.48 & $61.48^{* * *}$ \\
\hline \multicolumn{6}{|l|}{ Pig Latin } \\
\hline Accuracy $^{1}$ & 3.68 & 2.32 & 5.30 & 1.29 & $14.71^{* * * *}$ \\
\hline Reaction time ${ }^{2}$ & 44.24 & 15.47 & 27.18 & 9.14 & $35.37^{* * *}$ \\
\hline \multicolumn{6}{|l|}{ Spoonerisms } \\
\hline Accuracy $^{1}$ & 3.68 & 1.75 & 5.55 & 0.71 & $38.95^{* * *}$ \\
\hline Reaction time $^{2}$ & 71.95 & 36.28 & 30.50 & 10.98 & $47.53^{* * *}$ \\
\hline
\end{tabular}

Notes. ${ }^{* * *} p<0.01 ;{ }^{* * * *} p<0.001 ;{ }^{1}$ Number of correct responses; ${ }^{2}$ In seconds; ${ }^{3}$ Number of participants is smaller due to missing data.

Reading non-words (Shatil, 1995b). This task was designed to measure the amount of correct words per minute that the participant is able to read. The participant was presented with a list of 86 solitary dotted non-words, creating a high spelling-to-sound correspondence (Frost, 1994). Each participant was asked to read the words as fast and correct as he can during one minute. The score reflects the number of words per minute the participant read correctly (Shatil, 1995b).

Phoneme deletion (Share, 1997). Participants heard 20 Hebrew meaningful words and were instructed to repeat them while omitting one of the phonemes. The place of the omitted phoneme was changed (beginning, middle or end of word), creating different levels of difficulty in performing the task. Accuracy and reaction time were recorded.

Pig Latin (Shatil, 1995c). Participants heard six Hebrew non-words and were requested to repeat them while pronouncing the first phoneme at the end of the word and adding the vowel /a/. Accuracy and reaction time were recorded.

Spoonerisms (Shatil, 1995d). Participants heard six Hebrew pairs of words and were instructed to repeat them, while switching the first phoneme of the words. Accuracy and reaction time were recorded.

Backward digit span. Working memory was measured using the WAIS-III (Wechsler Adults Intelligence Scale version III) subtest for backward digit span. In this subtest, the experimenter reads aloud lists of digits at a rate of one digit per second. Immediately after the set of digits had been read, participants were instructed to report back the digits verbally in the reverse order they heard it. Participants received two trials at each set size starting at set size two and working up to set size eight. Testing was terminated when participants were incorrect on both trials of a given set size. The digit backward score was the number of digit backward trials where all digits were reported accurately in the correct reverse order (maximum score of 14) (Wechsler, 1997). Backward digit span is a subtask of the digit span subtest, and was used as an indicator for working memory (Gathercole \& Pickering, 2000). 


\section{Intensity Perception}

Absolute threshold. Absolute thresholds of the following tones were measured: $1 \mathrm{kHz}$ pure tone, $15 \mathrm{msec}$ duration; $1 \mathrm{kHz}$ pure tone, 50 msec duration; and $1.8 \mathrm{kHz}$ pure tone, $15 \mathrm{msec}$ duration, using a 2-alternative, forced-choice adaptive threshold paradigm and a two-down-one-up criterion (Grimault, Bacon, \& Micheyl, 2002; Levitt, 1971). Three blocks of stimuli were presented to each participant. The first block of stimuli was a practice run. Threshold was computed from the last two blocks. The threshold in each block was calculated as the mean of the last eight out of 10 reversals.

Intensity discrimination. On each trial, participants were presented with a pair of 500 msec duration, 1 $\mathrm{kHz}$ pure tones, separated by $100 \mathrm{msec}$. In each pair, one tone was presented at $40 \mathrm{~dB}$ above hearing level (40 $\mathrm{dB}$ ), and the other tone was presented 0.25 to $12 \mathrm{~dB}$ below $40 \mathrm{~dB}$. Participants were required to indicate whether the two tones in each pair were the same or different in intensity. Tone intensity deltas were $0.25,0.75$, 1.5, 2, 4, 6, 8, 10 and $12 \mathrm{~dB}$ and were presented randomly 16 times each, for a total of 144 trials. After every 32 trials, participants received a short recess. Percent correct was recorded for each participant for each delta intensity. The experimental session was preceded by a practice session in which stimulus pairs were presented with intensity deltas of $12 \mathrm{~dB}, 6 \mathrm{~dB}$ and $0 \mathrm{~dB}$. The practice stimulus pairs were repeated 16 times each. In the practice session, participants received feedback for each response. No feedback was provided during the experimental session. Threshold was computed as the intensity delta for $50 \%$ correct.

\section{Spectral Perception}

Spectral TOJ. On each trial, participants were presented with a pair of $15 \mathrm{msec}$ duration $1 \mathrm{kHz}$ (low) and $1.8 \mathrm{kHz}$ (high) tones, presented diotically at an intensity level of $40 \mathrm{~dB}$. Participants were required to indicate the order of presentation of the tones (high-low or low-high). Tone combinations were presented in random order with ISIs of 2, 5, 10, 15, 30, 60, 90, 120 and 240 msec. The order of the presentation of ISIs (inter-stimulus interval) was also random. Each ISI value was repeated 16 times, resulting in a total of 288 trials. After every 32 trials, participants received a short recess. Percent correct was recorded for each participant for each ISI.

To familiarize the participants with the tones, participants were first presented with six $1 \mathrm{kHz}$ (low) tones, then six $1.8 \mathrm{kHz}$ (high) tones. Training then proceeded with 24 trials, 12 to each tone, randomly intermixed. On each trial, the participant was required to identify the sound (low or high) by pressing the correct key. Then, the stimuli were presented again, with no feedback, until the participant met the criterion of 20 correct responses in 24 consecutive trials. Participants were then presented with 16 pairs of stimuli, with an ISI of 240 and 60 msec, for a total of 64 stimulus pairs. Participants were to identify which pattern they heard by pressing the key for the first sound followed by the key for the second sound. Visual feedback was provided on all training trials. No feedback was provided during the experimental session (Ben-Artzi et al., 2005). Threshold was computed as the ISI that was required for the participant to correctly reproduce the order of the tones in $75 \%$ of the trials ${ }^{1}$.

\section{Temporal Perception}

Gap detection. On each trial, subjects were presented with a pair of two 50 msec duration, $1 \mathrm{kHz}$ pure tones, separated by a 500 msec ISI. Each pair contained a target tone with a gap of silence that ranged between

\footnotetext{
${ }^{1}$ Only 25 participants (16 dyslexic readers and nine normal readers, $\chi_{(1)}^{2}=3.77, p=0.05$ ) had psychometric function eligible for calculating the threshold. Most of the participants' psychometric function was above 75\% (11 dyslexic readers and 25 normal readers, $\chi_{(1)}^{2}=5.44, p<0.05$ ), but for some of them, it did not reach it (10 dyslexic readers and six normal readers, $\chi^{2}(1)=1$, n.s.).
} 
$0.5 \mathrm{msec}$ to $36 \mathrm{msec}$, and a reference tone with a gap of $0 \mathrm{msec}$. This procedure of using a gap in both the reference and target tones was adapted in order to prevent judgments based on possible perceived changes in the overall envelope of a tone with a gap versus a tone without gap (Schneider et al., 1994). Subjects judged which of the two tones contained the gap. Gap durations were $0.5,1,2,4,8,12,18,24$ and 36 msec and were presented randomly 16 times each, for a total of 144 trials. After every 32 trials, subjects received a short recess. Percent correct was recorded for each participant for each ISI. The experimental session was preceded by a practice session participants received stimuli with 36 and 18 msec gaps that were repeated 16 times each. In practice sessions, subjects received feedback for each response. No feedback was provided during the experimental session. Threshold was computed as the gap that was required for correct detection in $75 \%$ of the trails.

Dichotic TOJ. On each trial, participants were presented with a pair of $15 \mathrm{msec}$ duration $1.8 \mathrm{kHz}$ tones, presented dichotically, i.e., the first tone to one ear the second tone to the other ear, at an intensity level of 40 dBSL. Participants were required to indicate the order of presentation of the tones (right-left or left-right). Tone combinations were presented in random order with ISIs of 5, 10, 15, 30, 60, 90, 120 and 240 msec. The order of the presentation of ISIs was also random. Each ISI value was repeated 16 times, resulting in a total of 256 trials. After every 32 trials, participants received a short recess. Percent correct was recorded for each participant for each ISI.

To familiarize the participants with the tones, participants were first presented with six tones to one ear, then six tones in the other tone. Training then proceeded with 24 trials, 12 to each ear, randomly intermixed. On each trial, the participant was required to identify the sound location by pressing the correct key. Then, the stimuli were presented again, with no feedback, until the participant met the criterion of 20 correct responses in 24 consecutive trials. Participants then presented with 16 pairs of stimuli, with an ISI of $240 \mathrm{msec}$ and $60 \mathrm{msec}$, for a total of 64 stimulus pairs. Participants were to identify which pattern they heard by pressing the key for the first sound followed by the key for the second sound. Visual feedback was provided on all training trials. No feedback was provided during the experimental session (Ben-Artzi et al., 2005). Threshold was computed as the ISI that was required for the participant to correctly reproduce the order of the tones in $75 \%$ of the trials.

\section{Apparatus}

Hearing sensitivity screening tests for all participants was performed using Danplex DA64 or Maico Hearing Instruments Ltd. MA32 audiometers.

All the psychophysical tasks were presented using a Pentium1 personal computer that controlled the stimulus presentation and recorded responses and response time. All of the auditory stimuli used in the various psychophysical tasks were generated by a sound-generator device (TDT-system II: Tucker-Davis Technologies, Gainesville, FL), and then presented binaurally through TDH-49 headphones. Tasks were programmed using Matlab $^{\mathrm{TM}}$ software version 6.5.

\section{Procedure}

The participants performed the study tests in random order, except for the absolute threshold task which was always measured first in order to determine the intensity of the stimuli to be presented in the other psychophysical tasks ( $40 \mathrm{~dB}$ ). Before entering the study, the participants were informed regarding the nature of the testing procedure, signed an informed consent and were tested by audiometer for hearing level screening. 
Participants were paid 300 NIS (New Israeli Shekel, equivalent to \$80 US). The study was approved by the Bar-Ilan University Institutional Review Board.

\section{Results}

Means, standard deviations and group differences statistics for all auditory processing tasks and working memory are presented in Table 2 .

Table 2

Means and SDs for Auditory Processing Tasks and Working Memory for Dyslexic and Normal Readers

\begin{tabular}{|c|c|c|c|c|c|}
\hline \multirow[t]{2}{*}{$\overline{\text { Task }}$} & \multicolumn{2}{|c|}{ Dyslexic readers } & \multicolumn{2}{|c|}{ Normal readers } & \multirow[t]{2}{*}{$F$} \\
\hline & Mean & $S D$ & Mean & $S D$ & \\
\hline $1 \mathrm{kHz} 15 \mathrm{msec}^{1}$ & 20.50 & 1.11 & 18.90 & 1.06 & 1.089 \\
\hline $1 \mathrm{kHz} 50 \mathrm{msec}^{1}$ & 12.28 & 1.23 & 12.48 & 1.18 & 0.013 \\
\hline $1.8 \mathrm{kHz} 15 \mathrm{msec}^{1}$ & 17.14 & 1.13 & 15.42 & 1.08 & 1.211 \\
\hline Intensity discrimination $^{1}$ & 4.75 & 0.25 & 4.36 & 0.24 & 1.280 \\
\hline Spectral TOJ ${ }^{2}$ & 133.25 & 74.5 & 45.72 & 39.76 & $10.59^{* *}$ \\
\hline Gap detection ${ }^{2}$ & 15.33 & 1.06 & 9.76 & 1.02 & $14.3^{* * *}$ \\
\hline Dichotic TOJ ${ }^{2}$ & 107.29 & 8.49 & 68.28 & 8.17 & $10.95^{* * *}$ \\
\hline Backward digit span ${ }^{3}$ & 6.51 & 1.95 & 8.23 & 2.41 & $8.23^{* *}$ \\
\hline
\end{tabular}

Notes. ${ }^{*} p<0.05 ;{ }^{* * *} p<0.01 ;{ }^{* * * *} p<0.001 ;{ }^{1} \mathrm{In} \mathrm{dB} ;{ }^{2}$ In msec; ${ }^{3}$ No. of correct trials.

\section{Intensity Processing Tasks}

Dyslexic and normal readers were compared on absolute threshold (hearing sensitivity) for $15 \mathrm{msec}$ duration, $1 \mathrm{kHz}$ tones; 50 msec duration, $1 \mathrm{kHz}$ tones; and $15 \mathrm{msec} 1.8 \mathrm{kHz}$ tones, and on supra-threshold intensity discrimination, using MANOVA (multivariate analysis of variance). No group effect was found for any of these dependent variables $\left(F_{(4,72)}=1.39\right.$, n.s., see Table 2$)$.

\section{Spectral Processing Task}

Since only 25 participants (16 dyslexic readers and 9 normal readers) had psychometric function eligible for calculating the threshold, the ANOVA (analysis of variance) performed only on these participants. A significant group effect was found for spectral TOJ thresholds (see Table 2). Spectral TOJ psychometric function for dyslexic and normal readers is shown in Figure 1.

\section{Temporal Processing Tasks}

Dyslexic and normal readers were compared on dichotic TOJ and gap detection thresholds, using MANOVA. Significant group effects were found for both tasks $\left(F_{(2,74)}=9.13, p<0.001\right.$, see Table 2). Dichotic TOJ and gap detection psychometric functions for dyslexic and normal readers are shown in Figures 2 and 3, respectively.

\section{Temporal and Spectral Processing Controlling for Working Memory}

Dyslexic readers had significantly lower scores on backward digit span than normal readers (see Table 2). As the number of participants in the spectral TOJ task was lower than in the other tasks, two separate MANCOVA were carried out between dyslexic and normal readers, one with the spectral TOJ task, and the other with dichotic TOJ and gap detection thresholds as dependent variables, and backward digit span as the covariate. Significant group effects were found both for spectral TOJ thresholds $\left(F_{(2,26)}=8.27, p<0.01\right)$ and 
for dichotic TOJ and gap detection thresholds $\left(F_{(2,73)}=6.95, p<0.01\right)$, even when controlling for backward digit span.

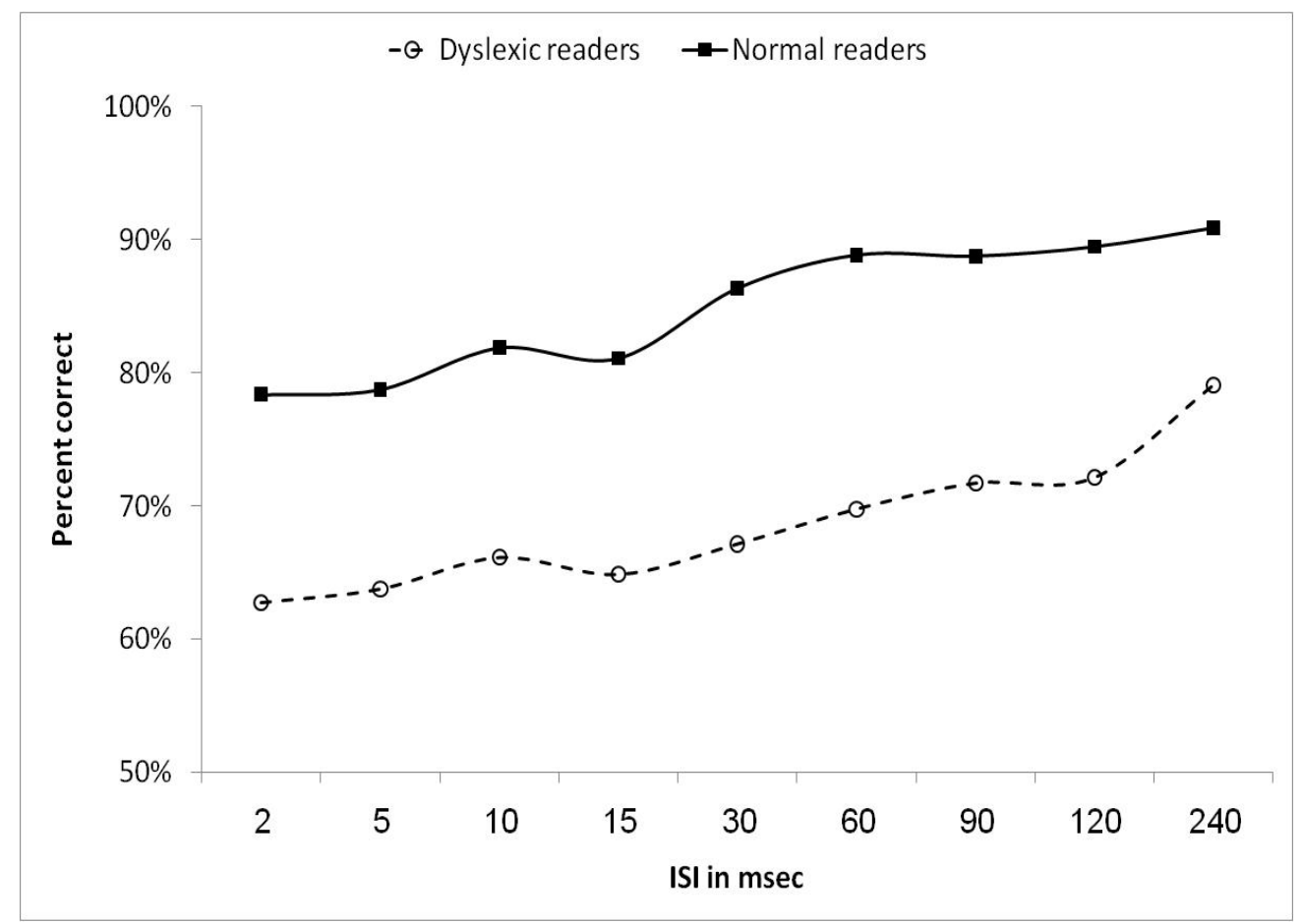

Figure 1. Mean percent correct by ISI for dyslexic and normal readers in spectral TOJ.

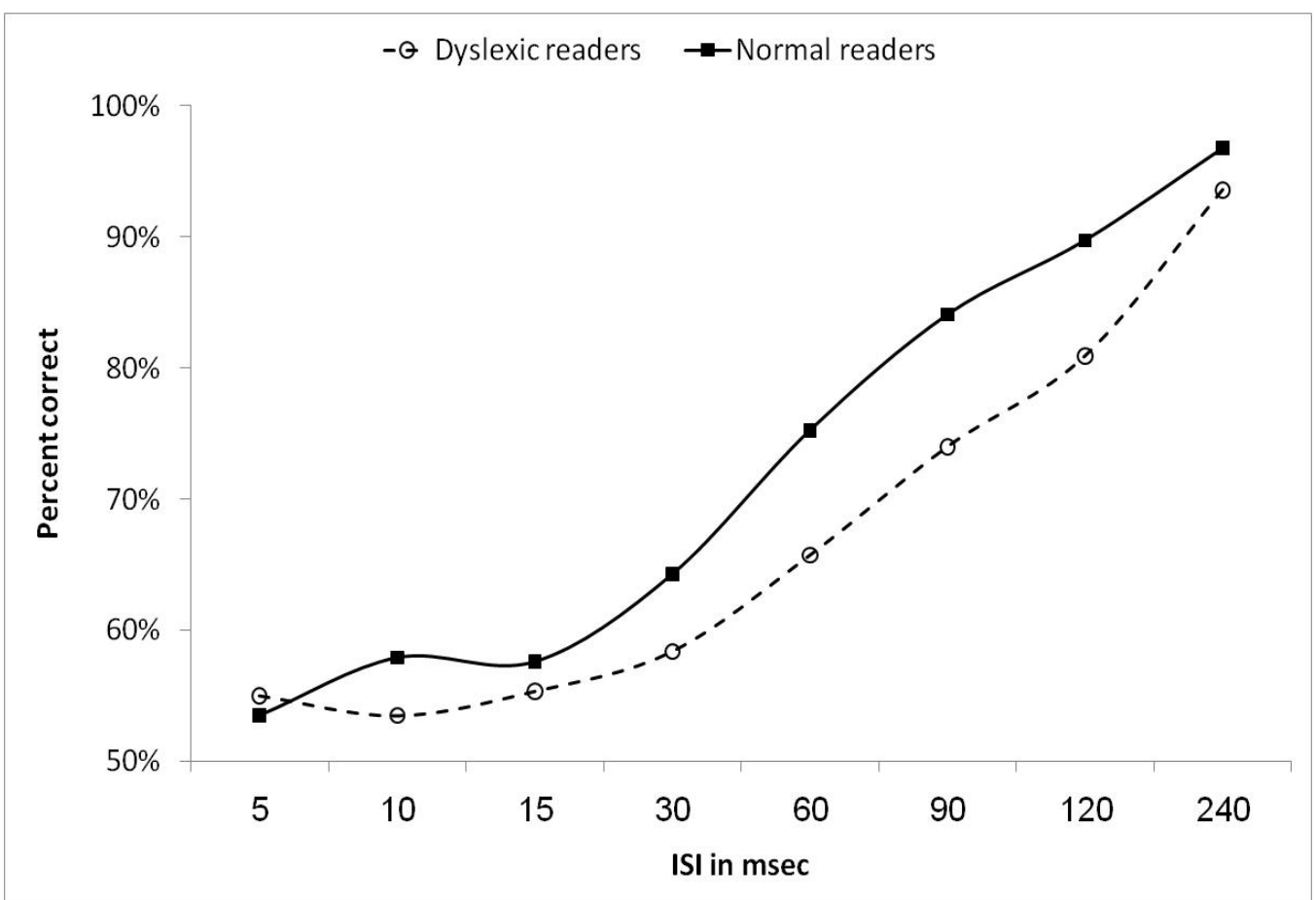

Figure 2. Mean percent correct by ISI for dyslexic and normal readers in dichotic TOJ. 


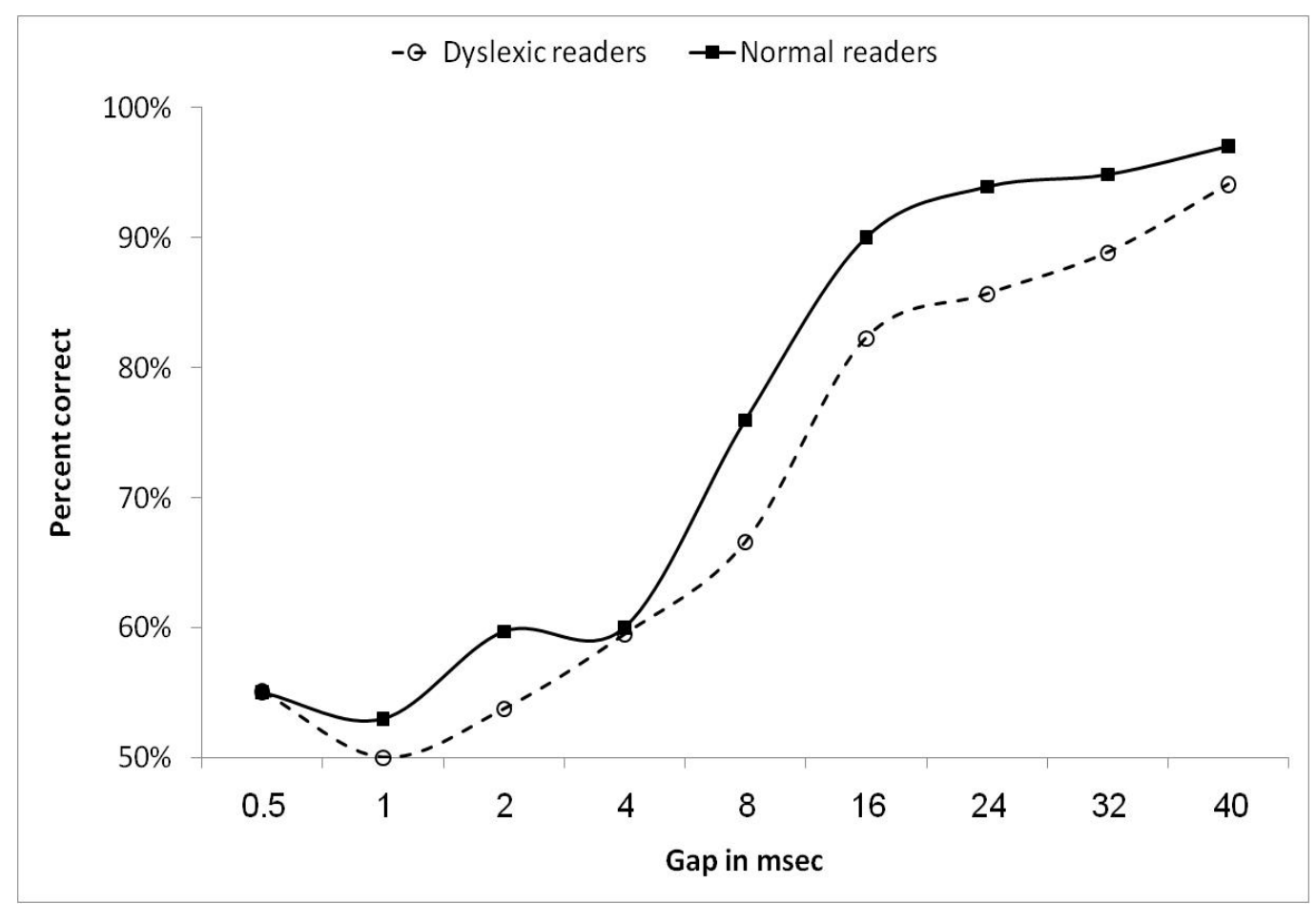

Figure 3. Mean percent correct by ISI for dyslexic and normal readers in gap detection.

\section{Discussion}

In the current study, we aimed to test whether auditory processing deficit will be evident when: (1) testing non-temporal processing; and (2) controlling for working memory. Performance on auditory spectral and temporal processing task was poorer among dyslexic readers, as compared to normal readers, but no difference was found in intensity processing tasks. Dyslexic readers' deficit on auditory temporal and spectral processing was evident even after controlling for working memory.

The data of the current study indicate that adult dyslexic readers exhibit deficit relative to the adult normal readers when the auditory discrimination is based on temporal manipulation, but not when auditory discrimination is based on manipulating stimulus intensity. These results complement a number of earlier findings which reported that adult dyslexics required longer ISIs to reproduce the order of two tones and longer silent periods within a tone to detect the presence of a gap, relative to the normal reader (Ben-Artzi et al., 2005; Chung et al., 2008; Schaffler, Sonntag, Hartnegg, \& Fischer, 2004; Tallal, 1980; Tallal \& Piercy, 1973a, 1973b; Reed, 1989; Van Ingelghem et al., 2001), and confirm the hypothesis that the main difficulty among dyslexic readers is in the processing of temporal-related auditory stimuli. These results suggest that dyslexic readers exhibit a specific deficit in auditory temporal processing and not a general perceptual deficit in auditory processing.

The findings on the spectral processing task pose a challenge. In accord with the literature, we found that dyslexic readers required longer ISIs in order to reproduce the order of the tones presented (Ben-Artzi et al., 2005; Chung et al., 2008; Reed, 1980; Tallal, 1989; Tallal \& Piercy, 1973a, 1973b). Others have also found poor performance among dyslexic readers on frequency discrimination tasks (Ahissar et al., 2000; Boets, Wouters, van Wieringen, \& Ghesquière, 2006; Cacace et al., 2000; Kujala et al., 2006). However, it is not clear whether these findings point to a deficit in temporal or spectral processing. As the performance on these tasks 
requires the integration of information over time, one might argue that poorer performance reflects auditory temporal processing deficit. In fact, a similar argument was raised regarding the early reports by Tallal and colleagues of auditory temporal deficit in dyslexic readers while using spectral TOJ tasks (Tallal's repetition tests, see Ben-Artzi et al., 2005; Chung et al., 2008; Reed, 1989; Tallal \& Piercy, 1973a, 1973b; Tallal, 1980). However, since the tones also differ spectrally, it might be argued that the difficulty in performing this task represents a deficit in spectral processing.

Dyslexic readers that participated in the study performed significantly more poorly on the working memory task. This finding is in line with studies showing poor performance in working memory tasks for dyslexic readers (Banai \& Ahissar, 2006; Brambati et al., 2006; Gathercole \& Pickering, 2000; Jeffries \& Everatt, 2004). In spite of the differences in working memory, the deficit in auditory temporal processing was evident even after controlling for differences in working memory, suggesting that the deficit in auditory processing is independent of difficulties in working memory. Consequently, the findings of the current study confirm our second hypothesis that dyslexic readers will perform poorly on tasks that require auditory temporal processing, independently from any deficit in working memory. Based on these findings, we conclude that the present study supports the hypothesis that dyslexic readers' deficit in auditory temporal processing is specific to temporal perception, and is not a product of a deficit in working memory, as it had been previously suggested (Ahissar, 2007; Banai \& Ahissar, 2004).

The comparable performance of dyslexic and normal readers on the intensity discrimination task is in accord with the findings of Ahissar et al. (2000) and Kujala et al. (2006) but is inconsistent with Cacace et al. (2000), Fischer and Hartnegg (2004) and McArthur and Hogben (2001) who found poorer performance of dyslexic readers on this task compared to normal readers. The discrepancy between the different findings might be related to various factors. Firstly, as it was suggested by Mengler, Hogben, Michie, and Bishop (2005), the studies that found differences in intensity discrimination between dyslexic and normal readers, have also shown large variability among reading impaired participants in their sensitivity to the intensity of the sounds. Consequently, they concluded that the results reporting deficit in auditory intensity processing among dyslexic children should be regarded with caution (Mengler et al., 2005). Secondly, the inconsistency between the findings could be explained through differences in the age of the participants tested. While the studies which reported no difference between dyslexic and normal readers tested young adults (Ahissar et al., 2000, pp. 18-58; Kujala et al., 2006, pp. 20-41), the studies who report on lower intensity discrimination among dyslexic readers tested children (Cacace et al., 2000, pp. 9-11; Fischer \& Hartnegg, 2004, pp. 7-22; McArthur \& Hogben, 2001, pp. 7-9). This might suggest that the specificity of the temporal processing deficit emerges in adulthood, when other perceptual processes mature. It is important to note that reading ability was poorer among dyslexic readers, both in the current study and in the previous studies that did not find differences in intensity discrimination (Ahissar et al., 2000; Kujala et al., 2006), suggesting that the presence of deficit in intensity discrimination ability might not be related to reading ability.

In conclusion, the findings of the present study provide further evidence of a specificity of auditory temporal processing among dyslexic readers, rather than a general auditory deficit. Moreover, the findings indicate that the deficit in auditory temporal processing is not a result of deficit in working memory.

\section{References}

Ahissar, M. (2007). Dyslexia and the anchoring-deficit hypothesis. Trends in Cognitive Sciences, 11(11), 458-465. 
Ahissar, M., Protopapas, A., Reid, M., \& Merzenich, M. M. (2000). Auditory processing parallels reading abilities in adults. Proceedings of the National Academy of Sciences of the United States of America, 97(12), 6832-6837.

Archibald, L. M., \& Gathercole, S. E. (2006). Short-term and working memory in specific language impairment. International Journal of Language and Communication Disorders, 41(6), 675-693.

Baddeley, A. (2003). Working memory and language: An overview. Journal of Communication Disorders, 36(3), $189-208$.

Baddeley, A., Gathercole, S., \& Papagno, C. (1998). The phonological loop as a language learning device. Psychological Review, 105(1), 158-173.

Banai, K., \& Ahissar, M. (2004). Poor frequency discrimination probes dyslexics with particularly impaired working memory. Audiol Neurootol, 9(6), 328-340.

Banai, K., \& Ahissar, M. (2006). Auditory processing deficits in dyslexia: Task or stimulus related? Cerebral Cortex, 16(12), 1718-1728.

Ben-Artzi, E., Fostick, L., \& Babkoff, H. (2005). Deficits in temporal-order judgments in dyslexia: Evidence from diotic stimuli differing spectrally and from dichotic stimuli differing only by perceived location. Neuropsychologia, 43, 714-723.

Boets, B., Wouters, J., van Wieringen, A., \& Ghesquière, P. (2006). Auditory temporal information processing in preschool children at family risk for dyslexia: Relations with phonological abilities and developing literacy skills. Brain and Language, 97(1), 64-79.

Brambati, S. M., Termine, C. ... Perania, D. (2006). Neuropsychological deficits and neural dysfunction in familial dyslexia. Brain and Research, 1113(1), 174-185.

Breier, J. I., Gray, L., Fletcher, J. M., Diehl, R. L., Klaas, P., Foorman, B. R., \& Molis, M. R. (2001). Perception of voice and tone onset time continua in children with dyslexia with and without attention deficit/hyperactivity disorder. Journal of Experimental Child Psychology, 80(3), 245-270.

Cacace, A. T., McFarland, D. J., Ouimet, J. R., Schrieber, E. J., \& Marro, P. (2000). Temporal processing deficits in remediation-resistant reading-impaired children. Audiology and Neuro-otology, 5(2), 83-97.

Chung, K. K., Ho, C. S., Chan, D. W., Tsang, S. M., \& Lee, S. H. (2010). Cognitive profiles of Chinese adolescents with dyslexia. Dyslexia, 16(1), 2-23.

Chung, K. K. H., McBride-Chang, C., Wong, S. W. L., Cheung, H., Penney, T. B., \& Ho, C. S. H. (2008). The role of visual and auditory temporal processing for Chinese children with developmental dyslexia. Annals of Dyslexia, 58, 15-35.

Curtin, S., Manis, F. R., \& Seidenberg, M. S. (2001). Parallels between the reading and spelling deficits of two subgroups of developmental dyslexia. Reading and Writing: An Interdisciplinary Journal, 14, 515-547.

De Clercq-Quaegebeur, M., Casalis, S., Lemaitre, M. P., Bourgois, B., Getto, M., \& Vallée, L. (2010). Neuropsychological profile on the WISC-IV of French children with dyslexia. Journal of Learning Disabilities, 43(6), 563-574.

Facoetti, A., Paganoni, P., Turatto, M., Marzola, V., \& Mascetti, G. G. (2000). Visual-spatial attention in developmental dyslexia. Cortex, 36(1), 109-123.

Fischer, B., \& Hartnegg, K. (2004). On the development of low-level auditory discrimination and deficits in dyslexia. Dyslexia, 10, 105-118.

Fostick, L., \& Babkoff, H. (2010). The perceptual cues for processing temporal order of tones differing by frequency or spatially (Ear). Presented at the 51st Annual Meeting of the Psychonomic Society. St. Louis, USA.

Frost, R. (1994). Prelexical and postlexical strategies in reading: Evidence from a deep and a shallow orthography. Journal of Experimental Psychology: Learning, Memory and Cognition, 20(1), 116-129.

Gathercole, S. E., \& Pickering, S. J. (2000). Working memory deficits in children with low achievements in the national curriculum at 7 years of age. British Journal of Educational Psychology, 70(Pt 2), 177-194.

Gathercole, S. E., Tiffany, C., Briscoe, J., \& Thorn, A. (2005). Developmental consequences of poor phonological short-term memory function in childhood: A longitudinal study. Journal of Child Psychology and Psychiatry, 46(6), 598-611.

Grimault, N., Bacon, S. P., \& Micheyl, C. (2002). Auditory stream segregation on the basis of amplitude-modulation rate. Journal of the Acoustical Society of America, 111, 1340-1348.

Jeffries, S., \& Everatt, J. (2004). Working memory: Its role in dyslexia and other specific learning difficulties. Dyslexia, 10(3), 196-214.

Kujala, T., Lovio, R., Lepistö, T., Laasonen, M., \& Näätänen, R. (2006). Evaluation of multi-attribute auditory discrimination in dyslexia with the mismatch negativity. Clinical Neurophysiology, 117, 885-893.

Laasonen, M., Leppämäki, S., Tani, P., \& Hokkanen, L. (2009). Adult dyslexia and attention deficit disorder in Finland—Project DyAdd. Journal of Learning Disabilities, 42(6), 511-527. 
Levitt, H. (1971). Transformed up-down methods in psychoacoustics. Journal of the Acoustical Society of America, 49(2), 467-477.

McArthur, G. M., \& Hogben, J. H. (2001). Auditory backward recognition masking in children with a specific language impairment and children with a specific reading disability. Journal of the Acoustical Society of America, 109(3), 1092-1100.

Mengler, E. D., Hogben, J. H, Michie, P., \& Bishop, D. V. (2005). Poor frequency discrimination is related to oral language disorder in children: A psychoacoustic study. Dyslexia, 11(3), 155-173.

Meyler, A., \& Breznitz, Z. (2005). Visual, auditory and cross-modal processing of linguistic and nonlinguistic temporal patterns among adult dyslexic readers. Dyslexia, 11(2), 93-115.

Murphy, C. F., \& Schochat, E. (2009). How auditory temporal processing deficits relate to dyslexia. Brazilian Journal of Medical and Biological Research, 42(7), 647-654.

Nagarajan, S., Mahncke, H., Salz, T., Tallal, P., Roberts, T., \& Merzenich, M. M. (1999). Cortical auditory signal processing in poor readers. Proceedings of the National Academy of Science of the USA, 96(11), 6483-6488.

Ram-Tsur, R., Faust, M., \& Zivotofsky, A. Z. (2008). Poor performance on serial visual tasks in persons with reading disabilities: Impaired working memory? Journal of Learning Disabilities, 41(5), 437-450.

Ramus, F. (2003). Developmental dyslexia: Specific phonological deficit or general sensorimotor dysfunction? Current Opinion in Neurobiology, 13, 212-218.

Ramus, F., Rosen, S., Dakin, S. C., Day, B. L., Castellote, J. M., White, S., \& Frith, U. (2003). Theories of developmental dyslexia: Insights from a multiple case study of dyslexic adults. Brain, 126(4), 841-865.

Reed, M. A. (1989). Speech perception and the discrimination of brief auditory cues in reading disabled children. Journal of Experimental Child Psychology, 48(2), 270-292.

Schäffler, T., Sonntag, J., Hartnegg, K., \& Fischer, B. (2004). The effect of practice on low-level auditory discrimination, phonological skills, and spelling in dyslexia. Dyslexia, 10(2), 119-130.

Serniclaes, W., Sprenger-Charolles, L., Carre, R., \& Demonet, J. F. (2001). Perceptual discrimination of speech sounds in developmental dyslexia. Journal of Speech Language and Hearing Research, 44(2), 384-399.

Share, D. L. (1997). Phoneme deletion (Unpublished test, University of Haifa).

Share, D. L., Jorm, A. F., MacLean, R., \& Matthews, R. (2002). Temporal processing and reading disability. Reading and Writing: An Interdisciplinary Journal, 15,151-178.

Shatil, E. (1995a). One-minute test for words (Unpublished test, University of Haifa).

Shatil, E. (1995b). One-minute test for pseudowords (Unpublished test, University of Haifa).

Shatil, E. (1995c). Pig Latin (Unpublished test, University of Haifa).

Shatil, E. (1995d). Spoonerism (Unpublished test, University of Haifa).

Snowling, M., Bishop, D. V., \& Stothard, S. E. (2000). Is preschool language impairment a risk factor for dyslexia in adolescence? Journal of Child Psychology and Psychiatry, 41(5), 587-600.

Stanovich, K. E. (1988). Science and learning disabilities. Journal of Learning Disabilities, 21(4), 210-214.

Stein, J. (2001). The magnocellular theory of developmental dyslexia. Dyslexia, 7, 12-36.

Swanson, H. L., \& Jerman, O. (2007). The influence of working memory on reading growth in subgroups of children with reading disabilities. Journal of Experimental Child Psychology, 96(4), 249-283.

Tallal, P. (1980). Auditory temporal perception, phonics, and reading disabilities in children. Brain Lang, 9(2), 182-198.

Tallal, P., \& Piercy, M. (1973a). Developmental aphasia: Impaired rate of non-verbal processing as a function of sensory modality. Neuropsychologia, 11(4), 389-398.

Tallal, P., \& Piercy, M. (1973b). Defects of non-verbal auditory perception in children with developmental aphasia. Nature, 241(5390), 468-469.

Van Ingelghem, M., van Wieringen, A., Wouters, J., Vandenbussche, E., Onghena, P., \& Ghesquiere, P. (2001). Psychophysical evidence for a general temporal processing deficit in children with dyslexia. Neuroreport, 12(16), 3603-3607.

Vellutino, F. R. (1979). The validity of perceptual deficit explanations of reading disability: A reply to Fletcher and Satz. Journal of Learning Disability, 12(3), 160-167.

Wechsler, D. (1997). Weschsler adult intelligence scale-III. San Antonio, T. X.: The Psychological Corporation. 